\title{
RANDOMIZED PROSPECTIVE STUDY ON TRAUMATIC PATELLAR DISLOCATION: CONSERVATIVE TREATMENT VERSUS RECONSTRUCTION OF THE MEDIAL PATELLOFEMORAL LIGAMENT USING THE PATELLAR TENDON, WITH A MINIMUM OF TWO YEARS OF FOLLOW-UP
}

\author{
Alexandre Carneiro Bitar ${ }^{1}$, Caio Oliveira D'Elia' ${ }^{1}$, Marco Kawamura Demange ${ }^{2}$, Alexandre Christo Viegas ${ }^{3}$, Gilberto Luis Camanho ${ }^{4}$
}

\begin{abstract}
Objective: The aim of this study was to compare the surgical results from reconstruction of the medial patellofemoral ligament (MPFL) with non-operative treatment of primary patellar dislocation. Methods: Thirty-nine patients (41 knees) with patellar dislocation were randomized into two groups. One group was treated conservatively (immobilization and physiotherapy) and other was treated surgically with reconstruction of the MPFL, and the results were evaluated with a minimum follow-up of two years. The Kujala questionnaire was applied to assess pain and quality of life, and recurrences were evaluated. Pearson's chi-square test and Fisher's exact test were used in the statistical evaluation. Results: The statistical analysis showed that the mean Kujala score was significantly lower in the conservative group (70.8),
\end{abstract}

compared with the mean value in the surgical group (88.9), with $\mathrm{p}=0.001$. The surgical group presented a higher percentage of "good/excellent" Kujala score results $(71.43 \%)$ than in the conservative group $(25.0 \%)$, with $p=0.003$. The conservative group presented a greater number of recurrences ( $35 \%$ of the cases), while in the surgical group there were no reports of recurrences and/or subluxation. Conclusions: Treatment with reconstruction of the medial patellofemoral ligament using the patellar tendon produced better results, based on the analysis of post-treatment recurrences and the better final results from the Kujala questionnaire after a minimum follow-up period of two years.

Keywords - Patellar Ligament; Knee; Reconstruction; Patellar Dislocation

\section{INTRODUCTION}

Acute patellar dislocation represents $2 \%$ to $3 \%$ of knee injuries, and is the second most common cause of traumatic hemarthrosis of the knee ${ }^{(1)}$.

Acute patellar dislocations can result in patellar instability in the long term, with recurrence rates of $15 \%$ to $44 \%$ after conservative treatment, pain, inability to return to sports (reported by up to $55 \%$ of patients) and patellofemoral arthrosis ${ }^{(2)}$.
Most first-time traumatic patellar dislocations were treated conservatively, except when there was associated patellar dislocation or osteochondral fractures of the lateral femoral condyle. However, studies that observed a recurrence rate of up to $44 \%$, findings of late symptoms such as previous knee pain and a recurrent instability rate above $50 \%$ after conservative treatment led to an increase in the initial treatment with surgical repair and reconstruction of the medial patellar stabilizers (medial patellofemoral ligament,

1 -Master's degree in Science from the Department of Orthopedics of the School of Medicine of Universidade de São Paulo; Orthopedist at Instituto Vita - São Paulo, SP, Brazil.

2 - Master's and Doctor's degree in Science from the Department of Orthopedics of the School of Medicine of Universidade de São Paulo; Assistant Physician of the Institute of Orthopedics and Traumatology of HC-FMUSP - São Paulo, SP, Brazil.

3 - Master's degree in Science from the Department of Orthopedics of the School of Medicine of Universidade de São Paulo; Orthopedist at Instituto Camanho and at Hospital do Coração - São Paulo, SP, Brazil.

4 - Full Professor of the Department of Orthopedics and Traumatology of the School of Medicine of Universidade de São Paulo - São Paulo, SP, Brazil. Study conducted at Vita Care and LIM 41 - Laboratory of Medical Investigation of the Musculoskeletal System of the Department of Orthopedics and Traumatology of the School of Medicine of Universidade de São Paulo.

Mailing address: Rua Mato Grosso, 306, $1^{\circ}$ andar, Higienópolis - 01239-040 - São Paulo, SP. Email: bitar@vita.org.br Study received for publication: $12 / 22 / 2010$, accepted for publication: $3 / 25 / 2011$.

The authors declare that there was no conflict of interest in conducting this work 
vastus medialis obliquus and medial retinaculum $)^{(1,3-5)}$.

Our understanding of the natural history of acute patellar dislocations has undergone significant changes recently, although the literature on this subject is still scarce. There are few randomized controlled studies $^{(6-8)}$ and only one systematic review ${ }^{(1)}$.

Conclusions based on results of existing studies should be reached with care due to the vast diversity of methodologies ${ }^{(1,7,9)}$.

As a result, there is a great deal of controversy regarding the ideal conduct in primary patellar dislocations.

The aim of this study was to analyze and to compare, prospectively and randomly, results obtained from two types of approach (reconstruction of the medial patellofemoral ligament with patellar tendon versus conservative approach) in the treatment of traumatic patellar dislocations, in relation to the $\mathrm{Ku}-$ jala score and to recurrence.

\section{MATERIAL AND METHODS}

The diagnosis of primary patellar dislocation was patellar displacement or was based on findings of a physical examination carried out by an experienced orthopedic surgeon (history of acute knee trauma, intra-articular hematoma, softening in the medial epicondyle and positive lateral patellar apprehension). All the patients had radiographies of the affected knee and the NMR scan was taken to detect potential chondral or osteochondral fragments and to confirm the ligament injury.

\section{Inclusion/exclusion criteria}

The inclusion criteria for this prospective study were:

1) Occurrence of acute lateral patellar dislocation up to three weeks before the treatment;

2) Absence of history of previous knee surgery or severe lesion in the affected knee;

3) Absence of coexisting severe tibiofemoral ligament lesion that requires repair;

4) Absence of large osteochondral fragments

(diameter $>15 \mathrm{~mm}$ ) that require fixation; and

5) Patient's authorization for participation in the study.

\section{Exclusion criteria}

1) Previous surgery on the injured knee;

2) Conditions associated with severe neuromuscular or congenital diseases;
3) Patients under 18 years of age;

4) Follow-up time under two years;

5) Patients with patellar dislocation or symptoms of patellar instability in the affected knee;

6) Non-traumatic patellar dislocation (e.g.: dislocation during gait or squatting with moderate stress on the knee, resulting in dislocation without acute pain in the knee);

7) Patients unable to following the treatment protocol.

From 2003 to 2006 we assessed 44 patients diagnosed with primary patellar dislocation. Two patients were excluded, as they presented osteochondral fragments (diameter $>15 \mathrm{~mm}$ ) that required fixation and three patients were lost in the follow-up (11.3\%).

Thirty-nine patients (41 knees) with acute patellar dislocation were randomized into two groups (conservative group and reconstruction group), and evaluated with minimum follow-up of two years (Figure 1).

One group (18 patients; 20 knees) received conservative treatment (physiotherapy) only, while the other group (21 patients; 21 knees) was treated surgically with reconstruction of the medial patellofemoral ligament (MPFL) with patellar tendon ${ }^{(10)}$.

In the conservative treatment group, the patients used an extension brace for three weeks followed by physiotherapy focusing on range of motion and quadriceps strengthening. During this period, the patients were started on isometric quadriceps exercises, as well as analgesia, cryotherapy and electrostimulation. The orthopedist executed passive mobilization of the knee during the patients' fortnightly visit. Weight bearing was allowed after three weeks. The exercises for gain of range of motion were increased in the third week, with the introduction of the stationary bicycle without load.

Initial proprioception and closed kinetic chain exercises were executed and gradually evolved into open chain exercises. The objective was to enable the patient to resume previous sports activities in approximately 16 to 24 weeks.

In the reconstruction group, we initiated the procedure arthroscopically to diagnose and treat possible cartilage lesions in all the patients ${ }^{(10)}$. The surgical incision started proximally at the level of the upper patellar margin and centrally between the medial patellar margin and the medial epicondyle. A descending incision was then made, directed toward the superomedial margin of the tibial tubercle. We separated the 
medial third of the patellar ligament (PL) from the rest of the ligament with dissection up to the patella. The PL was carefully detached from the patella up to the transition between the proximal third and medial third of the patella (Figure 2). We placed the stitches between the periosteum and the ligament using FiberWire absorbable threads (Arthrex, Naples, FL) to safely rotate the graft (Figure 3 ). The femoral insertion of the MPFL covered an area more posterior and proximal to the medial epicondyle (Figure 4).

A tunnel was then drilled using a bit of the same diameter as the graft, and this was secured with an absorbable interference screw (Figure 4) or with anchors (also absorbable or nonabsorbable). At the end, we sutured the distal edge of the vastus medialis muscle to the graft, which bestowed a dynamic component upon the reconstruction. No lateral release procedure was performed.

Rehabilitation started on the day of the patient's first return visit and the subject used an immobilizer for three weeks; isometric quadriceps exercises as well as analgesia, cryotherapy and electrostimulation were started during this period. The surgeon performed passive mobilization of the knee during the fortnightly visits and weight bearing was permitted immediately after surgery. The exercises for gain

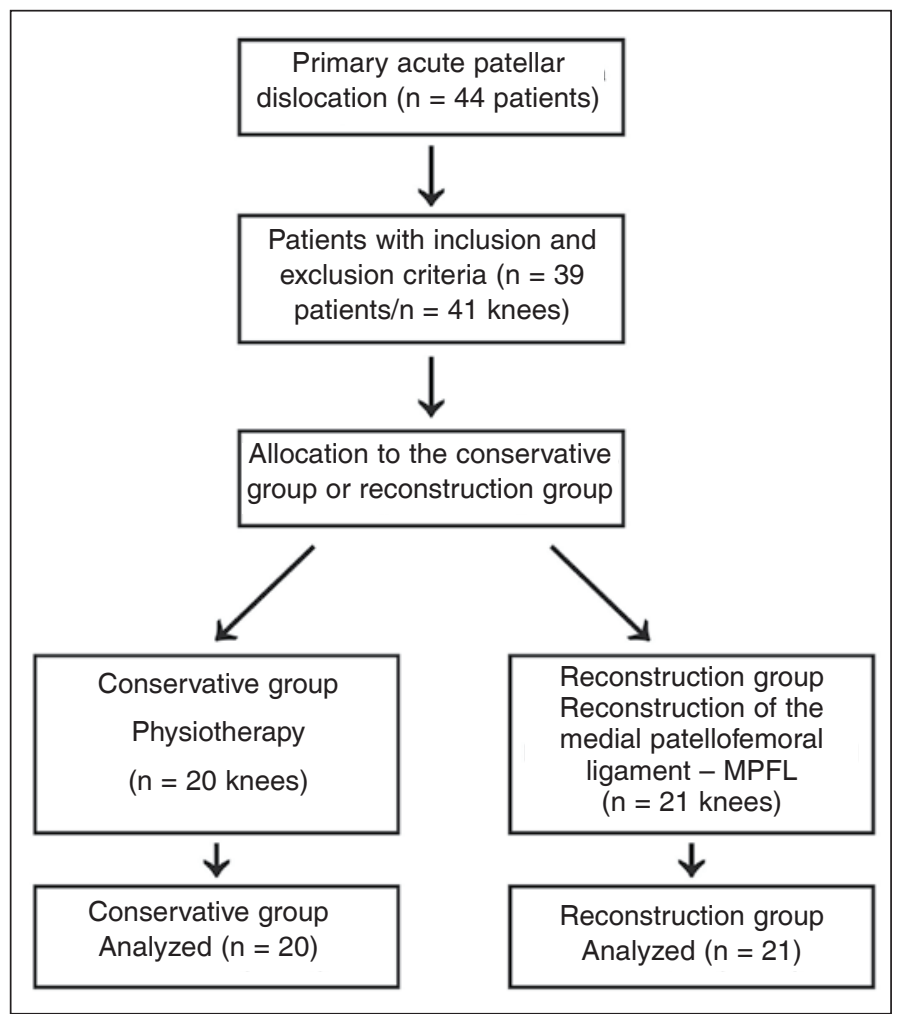

Figure 1 - Flowchart of the studied patients.

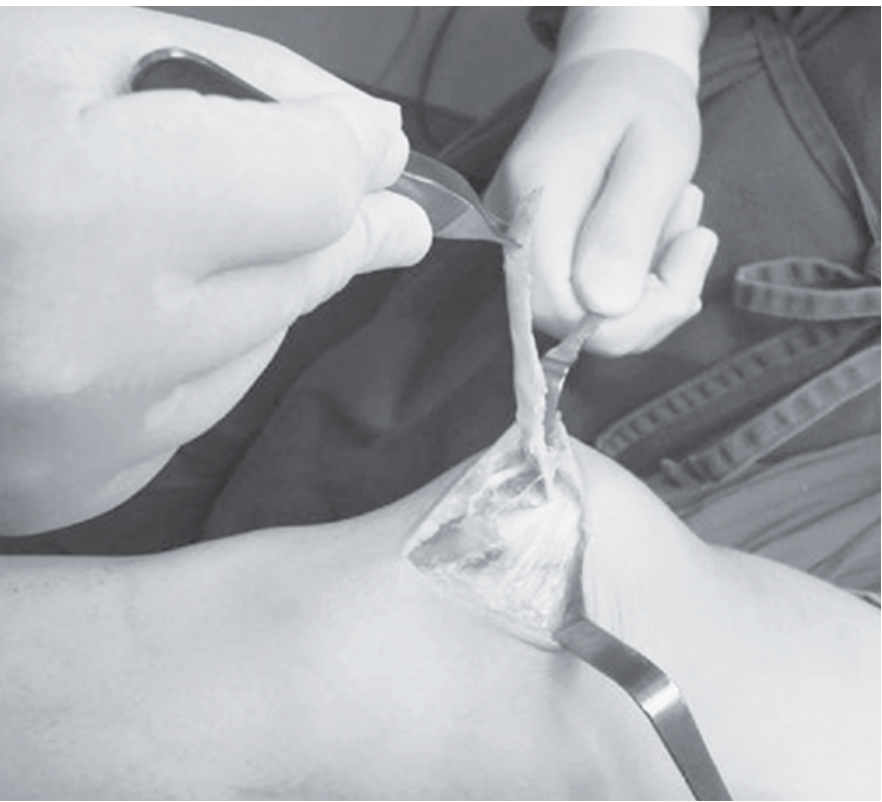

Figure 2-A $2 \mathrm{~cm}$ bone fragment is removed in order to detach the medial third of the patellar ligament from the rest of the ligament and to insert it in the patella.

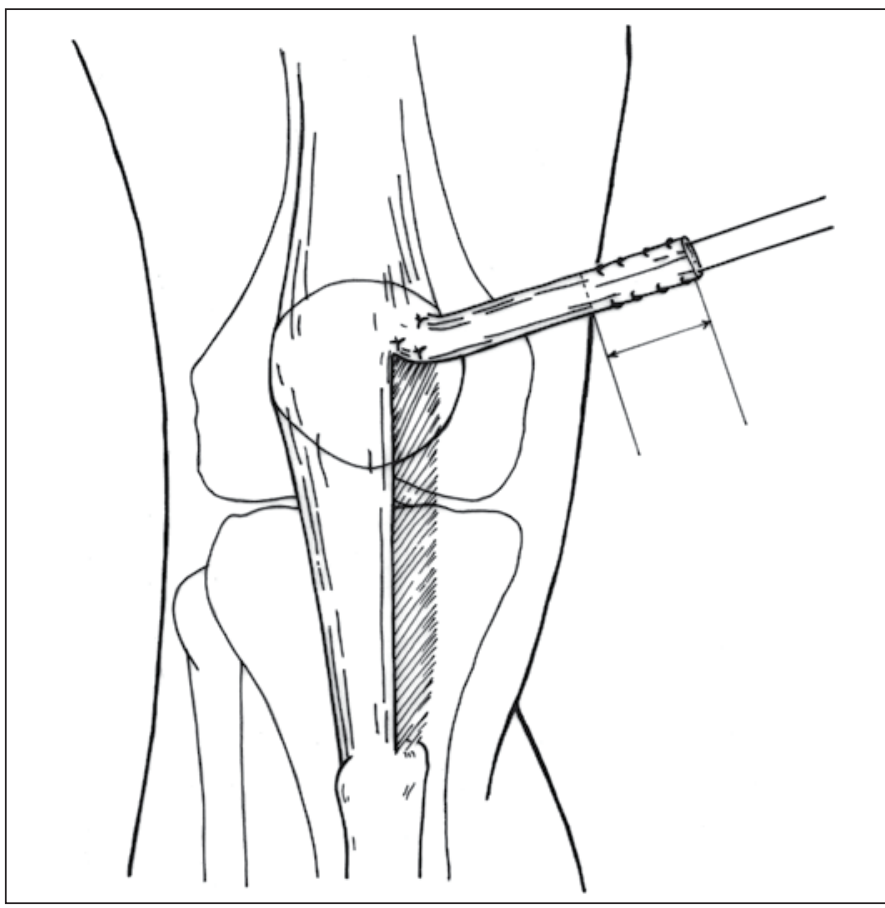

Figure 3 - Technique without the use of the bone fragment. Desinsertion is performed by planes up to the peritendon of the patellar ligament.

of range of motion were increased in the third week, with introduction of the stationary bicycle without load. Initial proprioception and closed kinetic chain exercises were executed and gradually evolved into open chain exercises. The objective was to enable the patient to resume previous sports activities in approximately 10 to 12 weeks. 


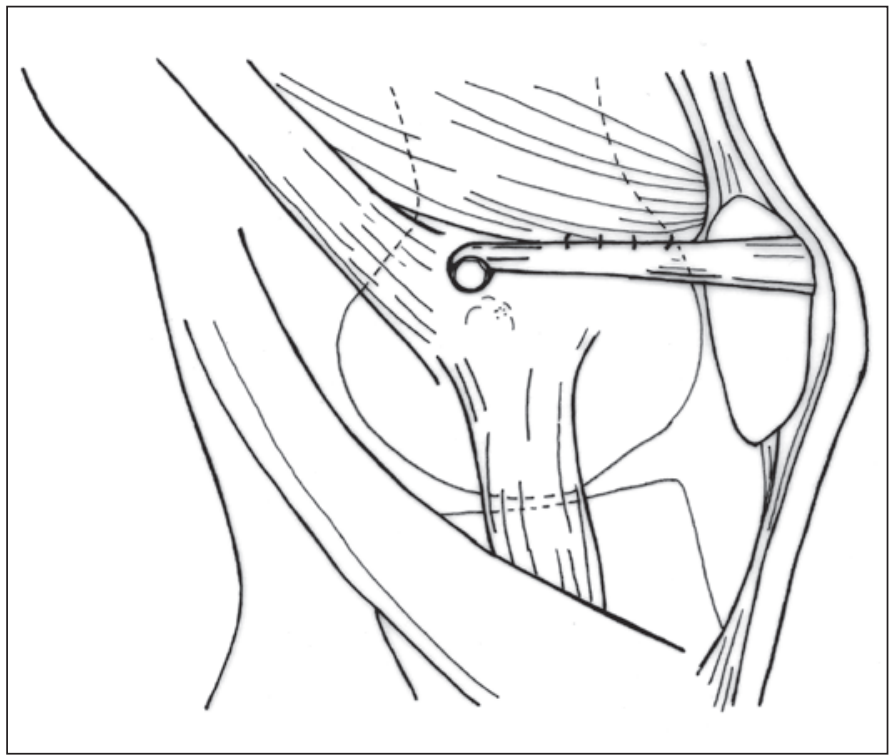

Figure 4 - Diagrammatic view of post-fixation of the medial structures, showing the site between the epicondyle and the adductor tubercles.

The Kujala questionnaire was applied to assess the patient's evolution in terms of pain and quality of life $\mathrm{f}^{(11)}$.

Recurrence was defined as a recurrent patellar dislocation that required a further visit to the physician or hospital. Patellar instability was defined as redislocation or subjective sensation of subluxation ${ }^{(7)}$.

The mean values of the Kujala score in the conservative and surgical groups were initially compared by means of a single factor (ANOVA). After this, still aiming to compare these mean values, we used the score adjusted to age, sex and side of knee (ANCOVA), since these factors can influence the comparison of interest.

The necessary assumptions for use of the proposed models were evaluated and proved appropriate for the data.

Besides the analysis of Kujala scores in their original form (continuous variable), we also conducted an analysis of this variable after being qualitatively categorized as: poor (Kujala $\leq 64$ ), fair (Kujala between 65 and 84) and good/excellent (Kujala $\geq 85$ ), as proposed by Sillanpaa et al $(2008)^{(12)}$.

The associations between the Kujala categories and the variables group, sex and side were evaluated by means of Pearson's chi-square test or Fisher's exact test, when the latter proved more adequate. A logistic regression model was used to estimate the odds ratio between Kujala (considering two categories - poor/fair and good/excellent) and group, both in a univariate manner and controlled by the covariables age, sex and side.

The need for the sample size to be 16 patients per study group was calculated with power of $90 \%$ and type I error (a) of 0.05 .

\section{RESULTS}

\section{Kujala Score}

Table 1 presents descriptive measures of the $\mathrm{Ku}$ jala score for each study group and also according to sex and affected side of knee. In general, the surgical group presented higher mean values in both knees and in both sexes. The scatter graph between the measures of the Kujala score and of the age of the patients in each group (Figure 5) did not indicate an association between these quantities, and Pearson's correlation coefficient was equal to 0.07 for the surgical group and -0.12 for the conservative group. We highlight the result of a low extreme value for the Kujala score (equal to 16), which corresponds to a measure of the conservative group, observed in a 25-year-old woman.

The statistical analysis showed that the mean $\mathrm{Ku}-$ jala score was significantly lower in the conservative group (70.8) when compared to the mean value of the surgical group (88.9), with $p=0.001$. This conclusion is maintained (with $p=0.002$ ) even after considering control by sex, age and side of the operated knee. Note that if we disregard the extreme observation emphasized earlier $($ Kujala $=16)$, the mean value of the conservative group rises from 70.8, with standard deviation (s.d.) of 19.2, to 73.7, with s.d. of 14.6. However, the removal of this value does not change the conclusions described previously, as the mean value of this group still appears significantly lower than that of the surgical group $(p<0.001)$, even after control by the covariables considered $(\mathrm{p}=0.001)$.

Table 2 presents the percentages of the Kujala score according to group, sex and side, considering the score divided into three or two categories (in this case, grouping poor and fair).

The analysis indicates association between group and Kujala score, while the surgical group presented a higher percentage of good/excellent results $(71.5 \%)$ when compared with the conservative group $(25.0 \%)$, with $\mathrm{p}=0.003$. It can also be seen that only $9.5 \%$ of the patients from the surgical group were classified as poor; while among those from the conservative group, this percentage was equal to $35.0 \%$. 
RANDOMIZED PROSPECTIVE STUDY ON TRAUMATIC PATELLAR DISLOCATION: CONSERVATIVE TREATMENT VERSUS RECONSTRUCTION OF THE MEDIAL PATELLOFEMORAL LIGAMENT USING THE PATELLAR TENDON, WITH A MINIMUM OF TWO YEARS OF FOLLOW-UP

Table 1 - Descriptive measures of the Kujala score for each study group according to sex and knee involved.

\begin{tabular}{|c|c|c|c|c|c|c|}
\hline \multirow[b]{2}{*}{ Group } & & \multicolumn{2}{|c|}{ Side of knee } & \multicolumn{2}{|c|}{ Sex } & \multirow[b]{2}{*}{ Total } \\
\hline & & Right & Left & Male & Female & \\
\hline \multirow[t]{6}{*}{ Reconstruction } & $\mathrm{N}$ & 9 & 12 & 9 & 12 & 21 \\
\hline & Mean & 92 & 86.5 & 85.2 & 91.6 & 88.9 \\
\hline & $\begin{array}{l}\text { Standard } \\
\text { Deviation }\end{array}$ & 6.8 & 12.2 & 13.5 & 6.7 & 10.4 \\
\hline & Median & 94 & 91 & 91 & 92 & 92 \\
\hline & Minimum & 82 & 62 & 62 & 82 & 62 \\
\hline & Maximum & 100 & 100 & 100 & 100 & 100 \\
\hline \multirow[t]{6}{*}{ Conservative } & $\mathrm{N}$ & 5 & 15 & 11 & 9 & 20 \\
\hline & Mean & 74 & 69.8 & 66.5 & 76.1 & 70.8 \\
\hline & $\begin{array}{l}\text { Standard } \\
\text { Deviation }\end{array}$ & 18.5 & 20 & 20.5 & 17.2 & 19.2 \\
\hline & Median & 83 & 71 & 71 & 83 & 73.5 \\
\hline & Minimum & 51 & 16 & 16 & 51 & 16 \\
\hline & Maximum & 94 & 96 & 87 & 96 & 96 \\
\hline \multirow[t]{6}{*}{ Total } & $\mathrm{N}$ & 16 & 27 & 20 & 21 & 41 \\
\hline & Mean & 85.6 & 77.2 & 75 & 84.9 & 80.1 \\
\hline & $\begin{array}{l}\text { Standard } \\
\text { Deviation }\end{array}$ & 14.6 & 18.7 & 19.7 & 14.3 & 17.7 \\
\hline & Median & 88.5 & 82 & 81 & 90 & 84 \\
\hline & Minimum & 51 & 16 & 16 & 51 & 16 \\
\hline & Maximum & 100 & 100 & 100 & 100 & 100 \\
\hline
\end{tabular}

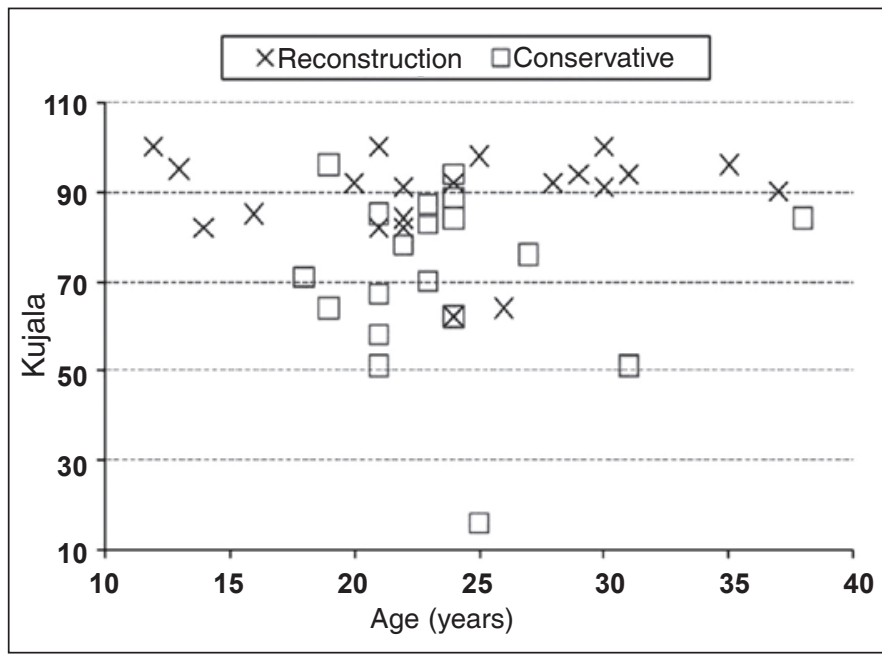

Figure 5 - Scatter graph between Kujala score and age of the patients, according to group.

In relation to the patients' age, Table 3 presents descriptive measures of this variable according to the categories of the Kujala score. In general, the distributions of ages do not present major differences, especially when the poor and fair categories are grouped.

Considering the division of the score into two categories, it is concluded that the odds of a patient
Table 2 - Distribution of the Kujala categories according to group, side involved and sex.

\begin{tabular}{|c|c|c|c|c|c|}
\hline & & \multicolumn{3}{|c|}{ Kujala } & \multirow[b]{2}{*}{ Tota } \\
\hline & & Poor & Fair & $\begin{array}{c}\text { Good/ } \\
\text { excellent }\end{array}$ & \\
\hline Group & Reconstruction & 2 & 4 & 15 & 21 \\
\hline$p^{1}=0.008$ & & $9.5 \%$ & $19.0 \%$ & $71.4 \%$ & $100 \%$ \\
\hline \multirow[t]{2}{*}{$\mathrm{p}^{2}=0.003$} & Conservative & 7 & 8 & 5 & 20 \\
\hline & & $35.0 \%$ & $40.0 \%$ & $25.0 \%$ & $100 \%$ \\
\hline Sex & Female & 7 & 5 & 8 & 20 \\
\hline$p^{1}=0.182$ & & 35.0 & 25.0 & $40.0 \%$ & $100 \%$ \\
\hline \multirow[t]{2}{*}{$\mathrm{p}^{2}=0.272$} & Male & 2 & 7 & 12 & 21 \\
\hline & & 9.5 & 33.3 & $57.1 \%$ & $100 \%$ \\
\hline $\begin{array}{c}\text { Side of } \\
\text { knee }\end{array}$ & Right & 2 & 4 & 8 & 14 \\
\hline$p^{1}=0.701$ & & $14.30 \%$ & $28.60 \%$ & $57.10 \%$ & $100 \%$ \\
\hline \multirow[t]{4}{*}{$\mathrm{p}^{2}=0.440$} & Left & 7 & 8 & 12 & 27 \\
\hline & & $25.90 \%$ & $29.60 \%$ & $44.40 \%$ & $100 \%$ \\
\hline & TOTAL & 9 & 12 & 20 & 41 \\
\hline & & $22.00 \%$ & $29.30 \%$ & $48.80 \%$ & $100 \%$ \\
\hline
\end{tabular}

${ }^{1} \mathrm{p}$-value referring to Fisher's exact test, with Kujala in three categories.

2p-value referring to Pearson's chi-square test, with Kujala in two categories: poor/ fair and good/excellent.

Table 3 - Descriptive measures of the patients' age according to the categories of the Kujala score.

\begin{tabular}{c|c|c|c|c|c|c|c}
\hline Kujala & Groups & $\mathbf{N}$ & Mean & $\begin{array}{c}\text { Standard } \\
\text { Deviation }\end{array}$ & Median & Minimum & Maximum \\
\hline $\begin{array}{c}\text { Poor/ } \\
\text { Fair }\end{array}$ & Conservative & 15 & 24.5 & 5.3 & 23 & 18 & 38 \\
\hline & Reconstruction & 6 & 21.5 & 4.1 & 22 & 14 & 26 \\
\hline & Total & 21 & 23.7 & 5.1 & 23 & 14 & 38 \\
\hline Good/ & Conservative & 5 & 22.2 & 2.2 & 23 & 19 & 24 \\
\hline & Reconstruction & 15 & 24.9 & 7.6 & 25 & 12 & 37 \\
\hline Total & Conservative & 20 & 24 & 4.8 & 23 & 18 & 38 \\
\hline & Reconstruction & 21 & 23.9 & 6.8 & 24 & 12 & 37 \\
\hline & Total & 41 & 23.9 & 5.8 & 23 & 12 & 38 \\
\hline
\end{tabular}


being classified as Kujala good/excellent are greater for those operated with the surgical method. The odds of a patient being classified as Kujala excellent/good in relation to fair/poor for those operated with the surgical method are 7.5 times the same odds for those operated with the conservative method, with a confidence interval of $95 \%$ ranging from 1.9 to 30.0 . This odds ratio also appears significantly different from 1 after control by the variables sex, side of knee affected and age of the patient (OR: 7.2; CI 95\%: 1.7 to 29.6).

\section{Recurrence and/or subluxation in the conserva- tive group}

In relation to cases of recurrence and/or subluxation in the conservative group, it was observed that of the 20 procedures evaluated, $35.0 \%$ (with confidence interval of $95 \%$ ranging from $14.1 \%$ to $55.9 \%$ ) exhibited recurrence or subluxation. It is also worth noting that of the two patients who had both sides affected, one of them presented recurrence on the right side and subluxation on the left side, and the other did not present any occurrence on either one of the sides.

\section{DISCUSSION}

There are many studies in relation to the surgical treatment of patellar dislocations with more than 100 surgical techniques, open or arthroscopic ${ }^{(1)}$.

There are few randomized controlled studies ${ }^{(6-8)}$ and only one systematic review ${ }^{(1)}$ comparing the conservative and surgical treatments.

In these studies, the authors recommend non-surgical treatment for primary patellar dislocations, except in cases where there is evidence of an osteochondral fragment. In case of the presence of an osteochondral fracture, arthroscopy is recommended for removal of the fragment or open repair according to its size. More specifically, the well-designed prospective, randomized study of Nikku et al $(1997)^{(8)}$ compared surgical treatment with conservative treatment in 125 patients with two years of follow-up. The results were evaluated subjectively by the patients' general opinion (excellent, good, reasonable and poor), by the Lysholm II score and by the visual analogue scale (VAS). The authors concluded that the surgical and conservative treatments produced almost identical results after two years in relation to the subjective scores, recurrence of instability and function. However, greater complica- tions only occurred after surgical treatment. Conclusions based on this study are hard to reach due to the reports of conservative treatment presented by Maenpaa and Lehto $(1997)^{(13)}$, which showed that more than half of the recurrences took place two years or more after the first dislocation.

In 2005, Nikku et $a l^{(7)}$ published their randomized, prospective study over a mean period of seven years with 125 patients. The study compared the conservative treatment, with immobilization and functional rehabilitation, with proximal realignment surgery (extensor mechanism realignment, repair of the medial patellar ligaments and/or lateral release). Their clinical results were very similar between the conservative and surgical groups. For this reason, Nikku et $a l^{(7)}$ do not recommend proximal realignment surgery for the treatment of primary patellar dislocations. This case series is a randomized, prospective, level I study, with long-term follow-up that compares the surgical conduct with the closed treatment of primary patellar dislocations. Moreover, episodes of redislocation and recurrent subluxation were included in a group together, and called episodes of instability, which contributed to the slightly higher recurrence rate in our series. We believe, like Stefancin and Parker $(2007)^{(1)}$, that the difference in surgical procedures makes it impossible to compare the two studies of Nikku et $a l^{(7)}$ and our study. As observed in many other surgical procedures, the results of the series of cases of MPFL repair appear to overestimate the success rates when compared with those of the randomized trials. Moreover, a wide range of different surgical procedures was carried out in the surgical group in the study conducted by Nikku et $a l^{(7)}$. Surgical intervention in $10 \%$ of the patients consisted of isolated lateral release. In an additional $81 \%$ of the patients, the medial retinaculum was repaired or duplicated, but the MPFL was not specifically used. Consequently, these results should not be extrapolated for procedures that involve repair or reconstruction of the MPFL. Finally, the trial of Nikku et $a l^{(7)}$ included both adults and children.

Another prospective and comparative, yet nonrandomized study, was that of Sillanpaa et $a l^{(12)}$. Our criticism consists of the fact that the magnetic resonance scan was only performed in the conservative group and the fixation of the surgical group was only performed in the patella. Another two comparative 
studies were those of Palmu et al ${ }^{(14)}$ and Buchner et $a l^{(9)}$. However, the first only studied patellofemoral dislocation in children and adolescents and the second is a retrospective study in which the authors excluded the patients with predisposing factors shown in the radiographies.

Finally, a recent randomized, prospective study was that of Christiansen $e t a l^{(6)}$. The main criticism of the study is that the patients from the surgical group were submitted to ligament repair on average 50 days after the traumatic episode and the lesion was fixed only at the femoral site.

We recently presented a nonrandomized prospective study comparing conservative treatment with MPFL repair ${ }^{(15)}$. In spite of the better rates of subjective scores and the lower number of recurrences of the surgical group in comparison to the conservative group, two patients presented subluxations with the same minimum follow-up time as our study on MPFL reconstruction.

Our study is the first randomized, prospective study comparing conservative treatment with a technique for reconstruction (and not repair) of the medial patellofemoral ligament (MPFL) described previously by Camanho et $a l^{(10)}$. We believe that MPFL reconstruction is the most appropriate form of surgical intervention for treating an acute traumatic patellar dislocation, since despite the controversy involving the site where the MPFL lesion occurs ${ }^{(5,12,16)}$, there are studies showing that intrasubstance injury of the MPFL is not infrequent ${ }^{(17)}$. In his article, Nomura ${ }^{(18)}$ showed the site of the MPFL injury through open exploration carried out on 27 knees after acute patellar dislocation. The MPFL suffered avulsion of its femoral origin in 10 knees, and one intrasubstance tear of the ligament was identified in another 16 knees. Sillanpaa et $a l^{(12)}$ observed an internal tear in the substance of the MPFL in 10 patients $(23 \%)$ in their study. In our clinical practice, the NMR often evidences a focal lesion on the femur or patella; however, we also observe signal alteration in the ligament in many cases, either due to a partial or total lesion, or just distension of the MPFL. This could indicate that repair and reinsertion of the MPFL at the AT, which is the insertion site of the MPFL, or failure in the identification and correction of the incompetence of the MPFL at the rupture site would compromise reasonable surgical treatment, which is also proposed by Christiansen et $a l^{(6)}$ and Hautamaa et $a l^{(19)}$, respectively.

Another aspect that we view as yet another advantage of reconstruction in relation to MPFL repair is the fact that it heals in an elongated manner, as proposed by Arendt et $a l^{(20)}$ in a review article of 2002.

Some case series present results that are also encouraging with the reconstruction of MPFL in the treatment of acute patellar dislocation ${ }^{(16)}$. The importance of associating techniques that recreate the anatomy and biomechanics of the MPFL is essential in surgery. Boden et $a l^{(21)}$ and Feller et $a l^{(22)}$ refer to the importance of the MPFL in the proprioception of the surrounding musculature. Through biomechanical studies, Amis et $a l^{(23)}$, Conlan et $a l^{(24)}$, Desio et $a l^{(25)}$, Sandmeier $e t a l^{(26)}$ and Tuxoe et $a l^{(27)}$ conclude that the MPFL is responsible for approximately 50 to $70 \%$ of the total lateral restriction, and is therefore, the primary medial stabilizer of the patella.

Another important aspect in our technique is that we also sewed the graft to the vastus medialis, introducing a dynamic component into the reconstruction. The direction of the fibers of the vastus medialis obliquus (VMO) and its anatomic relationship with the intramuscular septum proximally, and with the adductor magnus tendon distally, allow the VMO to traction the patella in oblique direction and thus to work as a dynamic medial stabilizer of the patella.

In relation to our technique, we believe that lateral release should never be executed in acute cases. According to literature, the role of lateral release is not clear when combined with surgery of medial patellar structures ${ }^{(3,12,24)}$. It was concluded that the performance of the lateral release in unstable patellae does not improve the clinical results, while this procedure can be beneficial for unstable and painful patellae.

The role of lateral release is not clear, but Desio et $a l^{(25)}$ suggested that lateral release may even increase lateral patellar instability, since their biomechanical study found that the lateral retinaculum contributes $10 \%$ to the retention strength. It is not known whether the lateral release performed in this study caused any effect on patellar instability. It was presumed that lateral release could cause additional instability in the hypermobile patellar joint. In this study, however, the dislocations were primary traumatic lesions and no hypermobile patellar joint was found ${ }^{(25,28-31)}$. 
Although recent review studies still consider conservative treatment the first option, some studies associate this treatment with evolution to recurrences ${ }^{(1,16)}$. In 1979, McManus et $a l^{(32)}$ published a study on the natural history of acute patellar dislocation. Recurrent dislocations occurred in $15 \%$ of the patients, with an additional $33 \%$ of patients experiencing sensations of instability or apprehension. Hawkins et al ${ }^{(33)}$ also gave an account of the natural history of acute patellar dislocation. Redislocation occurred in $15 \%$ of the patients, and a sensation of insecurity or knee instability persisted in another $20 \%$ of the patients. Maenpaa and Lehto ${ }^{(13)}$ monitored 100 patients treated nonoperatively after patellar dislocation for 13 years on average. Forty-four percent of the patients presented recurrence, and another 19\% presented recurrence of subluxation and patellofemoral pain.

Surprisingly, there is little evidence in relation to the conservative treatment of primary patellar dislocation $^{(2)}$. Treatment protocols range from immediate mobilization without the use of a brace to plaster cast immobilization in extension for six weeks. Immobilization in extension could provide the medial structures, particularly the MPFL, with a better means of achieving their healing. However, this entails the stiffness that frequently accompanies prolonged immobilization. The patient's adhesion can also be a factor in the decision for conservative treatment. For these reasons, many clinicians decide on a short period of immobilization followed by knee rehabilitation with or without a brace.

In our study, in the conservative treatment group, the patients remained in extension with the brace for three weeks followed by physiotherapy focused on range of motion and quadriceps strengthening.

Articular cartilage lesions are more common than previously believed and were reported in about $10 \%$ to $40 \%$ of the cases ${ }^{(9)}$; however, according to Nomu$\mathrm{ra}^{(18)}$, this incidence can be as high as $95 \%$ of primary patellar dislocations. What we take into account is the fragment size and the intraoperative evaluation of the need to fix the lesion. In our study, we excluded two patients with osteochondral fragments (diameter $>15$ $\mathrm{mm}$ ) that required fixation.

As regards our results, we consider the superiority of the results of the reconstruction group plainly obvious in comparison to the conservative group, both in relation to recurrence (and consequently instability) and in relation to the specific Kujala patellofemoral score.

As regards the Kujala score, our results upon quantitative analysis showed a clear advantage of the reconstruction group.

According to a study by Sillanpaa et $a l^{(12)}$, the $\mathrm{Ku}-$ jala score can be analyzed qualitatively: with a maximum possible score of 100 points (without symptoms), a rating of 95 points or more is excellent; 94 to 85, good; 84 to 65 , reasonable; and 64 or less, poor. Analyzing our sample this way, we still evidenced superiority of the surgical group in the results of this score.

Our study presents some points to be criticized: firstly due to the short follow-up time (in spite of the minimum period of two years) for us to evaluate mainly the recurrence rate. There is a tendency for redislocation with a longer follow-up time, both in the conservative and in the surgical series ${ }^{(13,34)}$. The short follow-up of the surgical series can explain the better results obtained after primary surgery.

The second criticism is our failure to separate the cases of patients with predisposing factors considered in literature. In this study we really did not analyze the predisposing factors. However, a recent study by our service clearly demonstrates that the predisposing factors did not influence the results ${ }^{(15)}$. As the reconstruction study followed the repair study we did not consider predisposing factors.

Finally, another criticism concerns the number of patients studied when compared with other studies in literature $^{(6-8)}$. However, we believe that our statistical analysis presented power of comparison in relation to the score used since it was necessary for the sample size to be at least 16 patients per study group and for this to have been calculated with power of $90 \%$, and type I error (a) of 0.05 .

\section{CONCLUSION}

We were able to conclude that the treatment with reconstruction of the medial patellofemoral ligament with the patellar tendon produced better results, based on the analyses of post-treatment recurrences and on the better end results of the Kujala questionnaire after a minimum follow-up of two years. 


\section{REFERENCES}

1. Stefancin JJ, Parker RD. First-time traumatic patellar dislocation: a systematic review. Clin Orthop Relat Res. 2007;455:93-101.

2. Mehta VM, Inoue M, Nomura E, Fithian DC. An algorithm guiding the evaluation and treatment of acute primary patellar dislocations. Sports Med Arthrosc. 2007;15(2):78-81.

3. Ahmad CS, Stein BE, Matuz D, Henry JH. Immediate surgical repair of the medial patellar stabilizers for acute patellar dislocation. A review of eight cases. Am J Sports Med. 2000;28(6):804-10.

4. Cash JD, Hughston JC. Treatment of acute patellar dislocation. Am J Sports Med. 1988;16(3):244-9.

5. Sallay PI, Poggi J, Speer KP, Garrett WE. Acute dislocation of the patella. A correlative pathoanatomic study. Am J Sports Med. 1996;24(1):52-60.

6. Christiansen SE, Jakobsen BW, Lund B, Lind M. Isolated repair of the medial patellofemoral ligament in primary dislocation of the patella: a prospective randomized study. Arthroscopy. 2008;24(8):881-7.

7. Nikku R, Nietosvaara $\mathrm{Y}$, Aalto K, Kallio PE. Operative treatment of primary patellar dislocation does not improve medium-term outcome: A 7-year follow-up report and risk analysis of 127 randomized patients. Acta Orthop. 2005;76(5):699-704.

8. Nikku R, Nietosvaara Y, Kallio PE, Aalto K, Michelsson JE. Operative versus closed treatment of primary dislocation of the patella. Similar 2-year results in 125 randomized patients. Acta Orthop Scand. 1997;68(5):419-23.

9. Buchner M, Baudendistel B, Sabo D, Schmitt H. Acute traumatic primary patellar dislocation: long-term results comparing conservative and surgical treatment. Clin J Sport Med. 2005;15(2):62-6.

10. Camanho GL, Bitar AC, Hernandez AJ, Olivi R. Medial patellofemoral ligament reconstruction: a novel technique using the patellar ligament. Arthroscopy. 2007;23(1):108 e101-4.

11. Kujala UM, Jaakkola LH, Koskinen SK, Taimela S, Hurme M, Nelimarkka O. Scoring of patellofemoral disorders. Arthroscopy. 1993;9(2):159-63.

12. Sillanpaa PJ, Maenpaa HM, Mattila VM, Visuri T, Pihlajamaki H. Arthroscopic surgery for primary traumatic patellar dislocation: a prospective, nonrandomized study comparing patients treated with and without acute arthroscopic stabilization with a median 7-year follow-up. Am J Sports Med. 2008;36(12):2301-9

13. Maenpaa H, Lehto MU. Patellar dislocation. The long-term results of nonoperative management in 100 patients. Am J Sports Med. 1997;25(2):213-7.

14. Palmu S, Kallio PE, Donell ST, Helenius I, Nietosvaara Y. Acute patellar dislocation in children and adolescents: a randomized clinical trial. J Bone Joint Surg Am. 2008;90(3):463-70.

15. Camanho GL, Viegas Ade C, Bitar AC, Demange MK, Hernandez AJ. Conservative versus surgical treatment for repair of the medial patellofemoral ligament in acute dislocations of the patella. Arthroscopy. 2009;25(6):620-5

16. Dopirak RM, Steensen RN, Maurus PB. The medial patellofemoral ligament. Orthopedics. 2008;31(4):331-8.
17. Elias DA, White LM, Fithian DC. Acute lateral patellar dislocation at MR imaging: injury patterns of medial patellar soft-tissue restraints and osteochondral injuries of the inferomedial patella. Radiology. 2002;225(3):736-43.

18. Nomura E. Classification of lesions of the medial patello-femoral ligament in patellar dislocation. Int Orthop. 1999;23(5):260-63.

19. Hautamaa PV, Fithian DC, Kaufman KR, Daniel DM, Pohlmeyer AM. Medial soft tissue restraints in lateral patellar instability and repair. Clin Orthop Relat Res. 1998(349):174-82.

20. Arendt EA, Fithian DC, Cohen E. Current concepts of lateral patella dislocation Clin Sports Med. 2002;21(3):499-519.

21. Boden BP, Pearsall AW, Garrett WE Jr, Feagin JA Jr. Patellofemoral instability: evaluation and management. J Am Acad Orthop Surg. 1997;5(1):47-57.

22. Feller JA, Feagin JA Jr, Garrett WE Jr. The medial patellofemoral ligament revisited: an anatomical study. Knee Surg Sports Traumatol Arthrosc. 1993;1(3-4):184-6.

23. Amis AA, Firer P, Mountney J, Senavongse W, Thomas NP. Anatomy and biomechanics of the medial patellofemoral ligament. Knee. 2003;10(3):215-220.

24. Conlan T, Garth WP Jr, Lemons JE. Evaluation of the medial soft-tissue restraints of the extensor mechanism of the knee. J Bone Joint Surg Am. 1993;75(5):682-93.

25. Desio SM, Burks RT, Bachus KN. Soft tissue restraints to lateral patellar translation in the human knee. Am J Sports Med. 1998;26(1):59-65.

26. Sandmeier RH, Burks RT, Bachus KN, Billings A. The effect of reconstruction of the medial patellofemoral ligament on patellar tracking. Am J Sports Med. May- 2000;28(3):345-9.

27. Tuxoe JI, Teir M, Winge S, Nielsen PL. The medial patellofemoral ligament: a dissection study. Knee Surg Sports Traumatol Arthrosc. 2002;10(3):138-40.

28. Fithian DC, Paxton EW, Post WR, Panni AS. Lateral retinacular release: a survey of the International Patellofemoral Study Group. Arthroscopy. 2004;20(5):463-8.

29. Jensen CM, Roosen JU. Acute traumatic dislocations of the patella. J Trauma 1985;25(2):160-2

30. Rosner B. Fundamentals of biostatistics. 6th ed. Belmont, CA: ThomsonBrooks/Cole; 2006.

31. Sherman OH, Fox JM, Sperling H, Del Pizzo W, Friedman MJ, Snyder SJ, et al. Patellar instability: treatment by arthroscopic electrosurgical lateral release. Arthroscopy. 1987;3(3):152-60.

32. McManus F, Rang M, Heslin DJ. Acute dislocation of the patella in children. The natural history. Clin Orthop Relat Res. 1979(139):88-91.

33. Hawkins RJ, Bell RH, Anisette G. Acute patellar dislocations. The natural history. Am J Sports Med. 1986;14(2):117-20.

34. Harilainen A, Sandelin J. Prospective long-term results of operative treatment in primary dislocation of the patella. Knee Surg Sports Traumatol Arthrosc. 1993;1(2):100-3. 